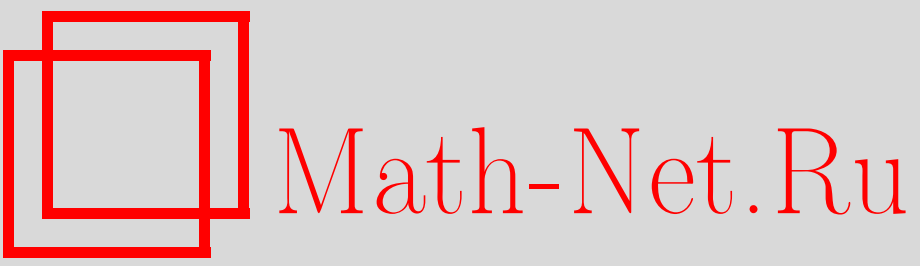

Ю. И. Кадашевич, С. П. Помыткин, Формулировка критерия прочности материалов для эндохронной теории неупругости, учитывающей микроразрушения при больших деформациях, Вестн. Сам. гос. техн. ун-та. Сер. Физ.-мат. науки, 2010, выпуск 1(), 53-59

DOI: https://doi.org/10.14498/vsgtu750

Использование Общероссийского математического портала Math-Net.Ru подразумевает, что вы прочитали и согласны с пользовательским соглашением

http://www.mathnet.ru/rus/agreement

Параметры загрузки:

IP : 54.147 .182 .235

26 апреля 2023 г., 03:24:40 
УДК 539.374, 535.422

\section{ФОРМУЛИРОВКА КРИТЕРИЯ ПРОЧНОСТИ МАТЕРИАЛОВ ДЛЯ ЭНДОХРОННОЙ ТЕОРИИ НЕУПРУГОСТИ, УЧИТЫВАЮШЕЙ МИКРОРАЗРУШЕНИЯ ПРИ БОЛЬШИХ ДЕФОРМАЦИЯХ}

\section{Ю. И. Кадашевич, С. П. Помыткин}

Санкт-Петербургский государственный технологический университет растительных полимеров, 198095, Санкт-Петербург, ул. Ивана Черных, 4.

E-mail: sppom@yandex.ru

Рассматриваются определяющие соотношения теории вязкопластичности эндохронного типа, распространенные на область больших дебормаций и поворотов. Предполагается, что в процессе дебормирования микроразрушения возникают как в упругих, так и в неупругих элементах материала. Предложены два определяющих уравнения и два закона дилатации для упругой и неупругой областей. Параметры разрыхления введены в критерий прочности для хрупкого и вязкого разрушения материалов.

Ключевые слова: неупругостъ, эндохронная теория, больиие деформации, определяющие соотношения, микроразрушения, критерий прочности.

Введение. Модель упруговязкопластичности, учитывающая микроразрушения в процессе неупругого деформирования материала, вводится в определяющие соотношения эндохронного типа, обобщённые на область больших деформаций и поворотов. Предполагается аддитивное представление тензора деформаций на упругую и неупругую составляющие. Причём считается, что микроразрушения в материале могут возникать как в упругой, так и в неупругой зонах деформирования, внося свой вклад в разрыхление и разрушение материала.

1. Критерий прочности Новожилова и его развитие. В работах $[1,2]$ был предложен критерий разрушения материалов, охватывающий как хрупкую, так и вязкую формы разрушения. Ответственность за разрушение материала возлагалась на пластическое разрыхление, мера которого $\theta$ и критерий определялись по формулам

$$
\begin{gathered}
\frac{d \theta}{d \lambda}=\beta, \quad d \lambda=\sqrt{d \varepsilon_{i j}^{p} d \varepsilon_{i j}^{p}}, \quad \theta=\varepsilon_{i i}^{p} \\
\theta=\theta^{*}
\end{gathered}
$$

где $\varepsilon_{i j}^{p}$ - тензор пластической деформации, $\lambda$ - параметр Одквиста, $\beta$ - константа материала, $\theta^{*}$ - разрыхление при разрушении.

В дальнейшем [3] выяснилось, что для лучшего согласования расчётов с опытными данными следует считать, что параметр $\beta$ не постоянен, а зависит от инварианта $\rho=\sqrt{\rho_{i j} \rho_{i j}}$, где $\rho_{i j}$ - тензор остаточных микронапряже-

Юлий Исаакович Кадашевич (д.ф.-м.н., проф.), профессор, каф. высшей математики. Сергей Павлович Помыткин (к.-ф.м.н., доц.), доцент, каф. высшей математики. 
ний [4]:

$$
\frac{d \theta}{d \lambda}=\beta(\rho) .
$$

В работе [5] было предложено более общее определяющее уравнение для параметра $\theta$ в форме

$$
\frac{d \theta}{d \lambda}=\beta(\rho, \theta) .
$$

Кроме того, отметим статьи [6,7], в которых предложены уравнения для разрыхления в виде

$$
\frac{d \theta}{d \lambda}=\beta(\lambda) \theta
$$

и

$$
\frac{d \theta}{d \lambda}=\beta\left(T_{0}\right), \quad T_{0}=\sigma_{0}-m \theta, \quad \sigma_{0}=\sigma_{i i}
$$

соответственно. И, наконец, в работе [8] в рамках эндохронного подхода предложено определяющее уравнение для обобщённого параметра разрыхления в форме

$$
\frac{d R_{0}}{d R}=\beta\left(R_{0}\right),
$$

где

$$
\begin{gathered}
d R=\sqrt{d R_{i j}^{\prime} d R_{i j}^{\prime}}, \quad R_{i j}^{\prime}=\varepsilon_{i j}^{\prime}-\frac{1-\alpha}{2 G} \sigma_{i j}^{\prime}, \\
R_{0}=\varepsilon_{0}-\frac{k_{1}}{K} \sigma_{0}, \quad \sigma_{0}=\sigma_{i i}, \quad \varepsilon_{0}=\varepsilon_{i i} .
\end{gathered}
$$

Здесь $\varepsilon_{i j}, \sigma_{i j}$ - тензоры деформаций и действующих напряжений, штрихами обозначены их девиаторы, $K$ - объёмный модуль, $G$ - модуль сдвига, $\alpha-$ параметр эндохронности, $k_{1}$ - константа материала.

Соотношения (1)-(7) относятся к случаю, когда скорость деформирования не играет роли. Поэтому авторы работ [9-11] рассмотрели более общий случай сложного нагружения, справедливый как для пластичности, так и для ползучести, то есть для достаточно общей теории необратимых процессов. Критерий разрушения, обобщающий формулу (3), выглядел следующим образом:

$$
\frac{d \theta}{d \lambda}=\rho \beta(\dot{\lambda}), \quad \theta_{k p}=m^{2}(\dot{\lambda}) .
$$

2. Учёт микроразрушений в неупругих элементах. В работе [12] при построении критерия прочности был использован простейший вариант эндохронной теории неупругости, учитывающий микроразрушения, когда разрушения наступают в упругих элементах. В данной статье приводится формулировка критерия прочности материалов для общего варианта эндохронной теории неупругости, учитывающей микроразрушения, когда в процессе деформирования микроразрушения происходят как в упругих, так и в неупругих элементах материала в условиях вязкопластичности при больших деформациях. 
Для этого, согласно идеям работы [13], сформулируем эндохронные определяющие соотношения двух типов - для упругих и неупругих составляющих:

$$
\begin{gathered}
\varepsilon_{i j}^{\prime e}+\alpha \rho_{1} \frac{d \varepsilon_{i j}^{\prime e}}{d R_{1}}=\rho_{1} \frac{d R_{i j}^{(1)}}{d R_{1}}+\frac{g_{1}+1}{g_{1}+\alpha} R_{i j}^{(1)}, \\
R_{i j}^{(1)}=\varepsilon_{i j}^{\prime e}-\frac{1-\alpha}{2 G_{1}} \sigma_{i j}^{\prime}, \quad d R_{1}=\sqrt{d R_{i j}^{(1)} d R_{i j}^{(1)}}, \quad \rho_{1}=\rho_{1}\left(R_{1}, \dot{R}_{1}\right) .
\end{gathered}
$$

Уравнения (8) можно записать и в следующем виде:

$$
2 G_{1}\left[\frac{\varepsilon_{i j}^{\prime e}}{g_{1}+\alpha}+\rho_{1} \frac{d \varepsilon_{i j}^{\prime e}}{d R_{1}}\right]=\rho_{1} \frac{d \sigma_{i j}^{\prime}}{d R_{1}}+\frac{g_{1}+1}{g_{1}+\alpha} \sigma_{i j}^{\prime}
$$

Здесь обозначены через $\varepsilon_{i j}^{\prime e}$ - девиатор тензора упругих деформаций, $\sigma_{i j}^{\prime}$ - девиатор тензора действующих (внешних) напряжений, $R_{i j}^{(1)}$ - девиатор вспомогательного параметрического тензора, $G_{1}$ - модуль сдвига, $\alpha$ - параметр эндохронности, $\rho_{1}$ - аналог предела упругого разрушения, $g_{1}$ - постоянный параметр материала.

Поступим аналогичным образом и для неупругих элементов модели:

$$
\begin{gathered}
\varepsilon_{i j}^{\prime p}+\beta \rho_{2} \frac{d \varepsilon_{i j}^{\prime p}}{d R_{2}}=\rho_{2} \frac{d R_{i j}^{(2)}}{d R_{2}}+\frac{g_{2}+1}{g_{2}+\beta} R_{i j}^{\prime(2)}, \\
R_{i j}^{\prime(2)}=\varepsilon_{i j}^{\prime p}-\frac{1-\beta}{2 G_{2}} s_{i j}^{\prime}, \quad d R_{2}=\sqrt{d R_{i j}^{(2)} d R_{i j}^{\prime(2)}}, \quad \rho_{2}=\rho_{2}\left(R_{2}, \dot{R}_{2}\right)
\end{gathered}
$$

или

$$
2 G_{2}\left[\frac{\varepsilon_{i j}^{\prime p}}{g_{2}+\beta}+\rho_{2} \frac{d \varepsilon_{i j}^{\prime p}}{d R_{2}}\right]=\rho_{2} \frac{d s_{i j}^{\prime}}{d R_{2}}+\frac{g_{2}+1}{g_{2}+\beta} s_{i j}^{\prime} .
$$

Здесь уже $\varepsilon_{i j}^{\prime p}$ - девиатор тензора неупругих деформаций, $s_{i j}^{\prime}$ - девиатор тензора микронапряжений, $R_{i j}^{(2)}$ - девиатор вспомогательного параметрического тензора, $G_{2}$ - модуль упрочнения, $\beta$ - параметр эндохронности, $\rho_{2}$ - аналог предела неупругого разрушения, $g_{2}$ - постоянный параметр материала.

При полностью активном нагружении выполняется равенство

$$
\varepsilon_{i j}^{\prime e}+\varepsilon_{i j}^{\prime p}=\varepsilon_{i j}^{\prime},
$$

где $\varepsilon_{i j}^{\prime}$ - девиатор тензора полных деформаций. Частный случай этих соотношений при $\rho_{1}=\rho_{1}\left(R_{1}\right)$ и $\rho_{2}=\rho_{2}\left(R_{2}\right)$ приведён в [14].

Если реализуется не полностью активное нагружение, то рекомендуем следующее дополнительное условие:

$$
s_{i j}^{\prime}=\sigma_{i j}^{\prime}-\tau\left(R_{1}, \dot{R}_{1}\right) \frac{d R_{i j}^{(1)}}{d R_{1}} .
$$


Если $\alpha=1$ и $\beta=1$, то, сохраняя эндохронность уравнений, легко получить более простые для анализа и восприятия определяющие соотношения в следующей форме:

$$
\begin{gathered}
2 G_{1}\left(\frac{\varepsilon_{i j}^{\prime e}}{g_{1}+1}+\rho_{1} \frac{d \varepsilon_{i j}^{\prime e}}{d \varepsilon_{1}^{e}}\right)=\rho_{1} \frac{d \sigma_{i j}^{\prime}}{d \varepsilon_{1}^{e}}+\sigma_{i j}^{\prime}, \\
2 G_{2}\left(\frac{\varepsilon_{i j}^{\prime p}}{g_{2}+1}+\rho_{2} \frac{d \varepsilon_{i j}^{\prime p}}{d \varepsilon_{1}^{p}}\right)=\rho_{2} \frac{d s_{i j}^{\prime}}{d \varepsilon_{1}^{p}}+s_{i j}^{\prime}, \\
d R_{1}=d \varepsilon_{1}^{e}=\sqrt{d \varepsilon_{i j}^{\prime e} d \varepsilon_{i j}^{\prime e}}, \quad d R_{2}=d \varepsilon_{1}^{p}=\sqrt{d \varepsilon_{i j}^{\prime p} d \varepsilon_{i j}^{\prime p}} .
\end{gathered}
$$

3. Определяющие соотношения и критерий прочности для больших деформаций. Указанные в п. 2 соотношения и уравнения записаны для области малых деформаций. При переходе к большим деформациям и большим поворотам поступим согласно рекомендациям, представленным, например, в работе [15]. Введём приведённые деформации и приведённые напряжения по формулам

$$
\begin{gathered}
E^{e}=Q^{\top} \varepsilon^{e} Q ; \quad E^{p}=Q^{\top} \varepsilon^{p} Q ; \quad T=Q^{\top} \sigma Q ; \\
N^{(1)}=Q^{\top} R^{(1)} Q ; \quad N^{(2)}=Q^{\top} R^{(2)} Q ; \\
\dot{E}^{e}=Q^{\top} D^{e} Q ; \quad \dot{E}^{p}=Q^{\top} D^{p} Q ; \quad \dot{T}=\overline{Q^{\top} \sigma Q} \\
\dot{N}^{(1)}=\overline{Q^{\top} R^{(1)} Q} ; \quad \dot{N}^{(2)}=\overline{Q^{\top} R^{(2)} Q} .
\end{gathered}
$$

Здесь и далее в безындексной форме записи тензоров будут использоваться следующие определения и соотношения: $Q$ - ортогональный тензор поворота, $D^{e}$ - тензор скоростей упругих деформаций, определяемый как $D^{e}=\left(L^{e}+\right.$ $\left.+L^{e \top}\right) / 2$, где $L^{e}=\dot{F}_{e} F_{e}^{-1}$, а $F_{e}=Q u_{e}$ - полярное разложение градиента упругой деформации $F_{e}$ и $u_{e}$-правый тензор упругого удлинения. Кроме того, введём упругий спин $W_{e}=\left(L^{e}-L^{e \top}\right) / 2$ и соотношение $u_{e}^{2}=F_{e}^{\top} F_{e}$.

Аналогично и для неупругих составляющих: $D^{p}$ - тензор скоростей неупругих деформаций, $D^{p}=\left(L^{p}+L^{p \top}\right) / 2, L^{p}=\dot{F}_{p} F_{p}^{-1}, F_{p}=Q u_{p}$ - полярное разложение градиента неупругой деформации $F_{p}, u_{p}$ - правый тензор неупругого удлинения, $W_{e}=\left(L^{e}-L^{e \top}\right) / 2-$ неупругий спин и $u_{p}^{2}=F_{p}^{\top} F_{p}$.

Фактически соотношения (13) и (14) определяют две меры деформацийупругой и неупругой соответственно:

$$
\varepsilon^{e}=Q\left(\int\left(Q^{\top} D^{e} Q\right) d t\right) Q^{\top}, \quad \varepsilon^{p}=Q\left(\int\left(Q^{\top} D^{p} Q\right) d t\right) Q^{\top} .
$$

С использованием определений (13), (14) соотношения (9), (11) примут вид

$$
\begin{aligned}
\left|\dot{R}_{1}\right| E^{e}+\alpha \rho_{1} \dot{E}^{e} & =\rho_{1} \dot{N}_{1}+\frac{g_{1}+1}{g_{1}+\alpha}\left|\dot{R}_{1}\right| N_{1}, \\
\left|\dot{R}_{2}\right| E^{p}+\beta \rho_{2} \dot{E}^{p} & =\rho_{2} \dot{N}_{2}+\frac{g_{2}+1}{g_{2}+\beta}\left|\dot{R}_{2}\right| N_{2} .
\end{aligned}
$$

Проведём над уравнениями (15) операцию обратного преобразования вида $Q(\cdot) Q^{\top}$, тогда получим, что 


$$
\begin{aligned}
\left|\dot{R}_{1}\right| \varepsilon^{e}+\alpha \rho_{1} \varepsilon^{e}=\rho_{1} \stackrel{\circ}{R}_{1}+\frac{\left|\dot{R}_{1}\right| R_{1}\left(g_{1}+1\right)}{g_{1}+\alpha}, \\
\left|\dot{R}_{2}\right| \varepsilon^{p}+\beta \rho_{2} \varepsilon^{p}=\rho_{2} \stackrel{\circ}{R}_{2}+\frac{\left|\dot{R}_{2}\right| R_{2}\left(g_{2}+1\right)}{g_{2}+\alpha},
\end{aligned}
$$

где кружок над тензором обозначает объективные производные типа ГринаНахгди:

$$
\begin{aligned}
& \stackrel{8}{\varepsilon}^{e}=\dot{\varepsilon}^{e}+\varepsilon^{e} \Omega-\Omega \varepsilon^{e}, \quad{ }^{p}{ }^{p}=\dot{\varepsilon}^{p}+\varepsilon^{p} \Omega-\Omega \varepsilon^{p}, \\
& \stackrel{\circ}{R}_{1}=\dot{R}_{1}+R_{1} \Omega-\Omega R_{1}, \quad \stackrel{\circ}{R}_{2}=\dot{R}_{2}+R_{2} \Omega-\Omega R_{2}
\end{aligned}
$$

и

$$
\Omega=\dot{Q} Q^{\top}, \quad \rho_{1}=\rho_{1}\left(R_{1}, \dot{R}_{1}\right), \quad \rho_{2}=\rho_{2}\left(R_{2}, \dot{R}_{2}\right) .
$$

Если воспользоваться соотношениями (8), (10), то уравнения (15) и (16) можно записать так:

$$
\begin{aligned}
& 2 G_{1}\left(\frac{\left|\dot{R}_{1}\right| E^{e}}{g_{1}+\alpha}+\rho_{1} \dot{E}^{e}\right)=\rho_{1} \dot{\sigma}+\frac{g_{1}+1}{g_{1}+\alpha}\left|\dot{R}_{1}\right| \sigma, \\
& 2 G_{2}\left(\frac{\left|\dot{R}_{2}\right| E^{p}}{g_{2}+\beta}+\rho_{2} \dot{E}^{p}\right)=\rho_{2} \dot{s}+\frac{g_{2}+1}{g_{2}+\beta}\left|\dot{R}_{2}\right| s
\end{aligned}
$$

и

$$
\begin{aligned}
& 2 G_{1}\left(\frac{\left|\dot{R}_{1}\right| \varepsilon^{e}}{g_{1}+\alpha}+\rho_{1} \varepsilon^{e}\right)=\rho_{1} \sigma^{\circ}+\frac{g_{1}+1}{g_{1}+\alpha}\left|\dot{R}_{1}\right| \sigma, \\
& 2 G_{2}\left(\frac{\left|\dot{R}_{2}\right| \varepsilon^{p}}{g_{2}+\beta}+\rho_{2} \varepsilon^{p} \varepsilon^{p}\right)=\rho_{2} \stackrel{\circ}{s}+\frac{g_{2}+1}{g_{2}+\beta}\left|\dot{R}_{2}\right| s .
\end{aligned}
$$

Эти соотношения - аналог уравнений (9) и (11), но уже для области больших деформаций и поворотов.

Если $\alpha=1, \beta=1, \rho_{1}=$ const, $\rho_{2}=$ const, то в одноосном случае из уравнений (17) реализуются следующие решения:

$$
\begin{gathered}
\sigma_{1}=\frac{2 G_{1}}{g_{1}+1}\left[\varepsilon_{1}^{e}+g_{1} \rho_{1}\left(1-\exp \left(-\varepsilon_{1}^{e} / \rho_{1}\right)\right)\right], \\
s_{1}=\frac{2 G_{2}}{g_{2}+1}\left[\varepsilon_{1}^{p}+g_{2} \rho_{2}\left(1-\exp \left(-\varepsilon_{1}^{p} / \rho_{2}\right)\right)\right], \\
d R_{1}=d \varepsilon_{1}^{e}, \quad d R_{2}=d \varepsilon_{1}^{p},
\end{gathered}
$$

а связь $\sigma_{1}$ с $s_{1}$ определяется формулой (13), а именно $s_{1}=\sigma_{1}-\tau_{1}\left(R_{1}, R_{2}\right)$.

Как было указано в п. $\mathbf{1}$, один из основных законов дилатации имеет вид

$$
\frac{d R_{0}}{d R}=\beta\left(R_{0}\right), \quad R_{0}=\varepsilon_{0}-\frac{k_{1}}{K} \sigma_{0} .
$$

В настоящей работе авторами рекомендуются, следуя идеям работ $[9,10]$, следующие законы дилатации и критерий:

$$
\frac{d R_{0}^{(1)}}{d R_{1}}=\beta_{1}\left(R_{0}^{(1)}, \sigma_{\star}, \dot{R}_{1}\right), \quad \sigma_{\star}=\sqrt{\sigma_{i j}^{\prime} \sigma_{i j}^{\prime}},
$$




$$
\begin{gathered}
\frac{d R_{0}^{(2)}}{d R_{2}}=\beta_{2}\left(R_{0}^{(2)}, s_{\star}, \dot{R}_{2}\right), \quad s_{\star}=\sqrt{s_{i j}^{\prime} s_{i j}^{\prime}}, \\
R_{0}=R_{0}^{(1)}+R_{0}^{(2)}=R_{0}^{*}\left(\dot{R}_{1}, \dot{R}_{2}\right) .
\end{gathered}
$$

4. Оценка критерия. В качестве примера рассмотрим ступенчатое нагружение при ползучести. Многочисленные экспериментальные наблюдения (см., например, [16-18]) показали, что принцип линейного суммирования не выполняется. Наиболее интересный закон остаточной долговечности был предложен Вудфордом [17]:

$$
\sum_{i=1}^{n} \Psi_{i}\left(t_{i} / t_{i}^{*}\right)=1,
$$

где $t_{i}$ - время действия нагружения на $i$-той ступени, $t_{i}^{*}$ - время до разрушения при нагружении на $i$-той ступени, а функции $\Psi_{i}$ находились экспериментально, причём при низких напряжениях эти функции выпуклы, а при больших напряжениях - вогнуты. Общий вывод из анализа экспериментальных данных таков: понижение напряжений увеличивает долговечность, а повышение напряжений ведёт к уменьшению долговечности. Критерий разрушения, предложенный в $[9,10]$, хорошо описывал отклонение от принципа линейного суммирования. Расчёты, проведённые по предлагаемому в данной работе варианту теории, оказались близкими к результатам работ $[9,10]$.

Авторами подробно была рассмотрена и задача простого сдвига. Эта задача представляет особый интерес, так как по классическим линейным теориям неупругости и по предложениям работы [9] осевые деформации не возникают, и использование критерия прочности, связанного с разрыхлением, бессмысленно. А по предлагаемой в данной работе теории при сдвиговых нагружениях возникают осевые деформации (небольшие по величине), а следовательно, имеет место и разрыхление материала. Результаты оказались вполне приемлемыми для практики.

Работа выполнена при поддержке РФФИ (код проекта 08-01-00036-а).

БИБЛИОГРАФИЧЕСКИЙ СПИСОК

1. Новожилов В. В. О пластическом разрыхлении // ПММ, 1965. - Т. 29, № 4. - С. 681689.

2. Новожилов В.В., Ръбакина О.Г. Перспективы построения критерия прочности при сложном нагружении // Инженерный журнал. МTT, 1966. - № 5. - С. 103-111.

3. Рыбакина О.Г. Феноменологическое описание разрушения металлов при некоторых видах асимметричного деформирования // Извест. АН CССР. MTT, 1969. - № 6. C. $61-66$.

4. Кадашевич Ю. И., Новожилов В. В. Теория пластичности, учитывающая эффект Баушингера // ДАН СССР, 1957. - Т. 117, № 4. - С. 586-588.

5. Бахвалова Н. А. Об учёте влияния накопления повреждённости на процесс разрушения в области малоцикловой усталости // Извест. АН CCCP. MTT, 1973. - № 2. - C. 143147.

6. Арутюнян Р. А. Об учёте эффекта Баушингера и объёмной пластической деформации в теории пластичности // Исследования по упругости и пластичности, 1968. - № 7. C. $53-61$.

7. Кадашевич Ю. И. Теория пластичности, учитывающая эффект Баушингера и влияние среднего нормального напряжения на границу текучести // Tp. Ленинград. технологич. ин-та иеллюлозно-бумажной пром-ти, 1965. - № 18. - С. 234-235.

8. Кадашевич Ю. И., Помыткин С. П. Описание трёхосного простого нагружения в рамках эндохронной теории неупругости, учитывающей дилатацию материалов / В сб.: Машины и аппараты иеллюлозно-бумажной промышленности, 2008. - С. 68-74. 
9. Новожилов В. В., Кадашевич Ю. И., Рыбакина О. Г. Разрыхление и критерий разрушения в условиях ползучести // ДАН СССР, 1983. - Т. 270, № 4. - С. 831-836.

10. Новожилов В. В., Кадашевич Ю.И., Рыбакина О. Г. Разрыхление и перспективы построения критерия прочности при сложном нагружении с учетом ползучести // Извест. АН CCCP. MTT, 1988. - № 5. - C. 108-114.

11. Кадашевич Ю. И., Новожилов В. В. Теория пластичности и ползучести металлов, учитывающая микронапряжения // Извест. АН СССР. МTT, 1981. - № 5. - С. 100-110.

12. Кадашевич Ю.И., Помыткин С. П. О построении критерия прочности при сложном нагружении / В сб.: Материаль 46-й международн. конф-иии. Ч. І. (Витебск, 1517 октября, 2007) / Актуальные проблемы прочности, 2007. - С. 68-74.

13. Кадашевич Ю.И., Помыткин С.П. О взаимосвязи теории пластичности, учитывающей микронапряжения, с эндохронной теорией пластичности // Извест. PAH. MTT, 1997. - № 4. - С. 99-105.

14. Кадашевич Ю. И., Помыткин С. П. Эндохронная теория неупругости, учитывающая микроразрушения (общий случай) / В сб.: Тр. 47-й международн. конф-иии. Ч. І. (Нижний Новгород, 1-5 июля, 2008) / Актуальные проблемы прочности, 2008. - С. 323324.

15. Кадашевич Ю. И., Помыткин С. П. Анализ сложного нагружения при конечных деформациях по эндохронной теории неупругости // Прикладные проблемы прочности и пластичности, 1998. - № 59. - С. 72-76.

16. Greenwood G.W. Fracture under creep conditions // Materials Science and Engineering, 1976. - Vol. 25. - P. 241-243.

17. Woodford D. A. Creep Damage and the Remaining Life Concept// ASME J. Eng. Mat. Technol., 1979. — Vol. 101, No.4. — P. 311-316; русск. пер.: Вудфорд Д. А. Повреждение при ползучести и концепция остаточной долговечности // Теоретические основы инженерных расчётов, 1979. - Т. 101, № 4. - С. 1-8.

18. Dyson B.F., McLean D. A New Method of Predicting Creep Life // Metal Science, 1972. Vol. 6, No. 1. - P. 220-223(4).

Поступила в редакцию 30/XII/2009;

в окончательном варианте - 15/III/2010.

MSC: 74C20

\section{FORMULATION OF MATERIAL STRENGTH CONDITION FOR ENDOCHRONIC THEORY OF NON-ELASTICITY ALLOWING FOR MICRO-FRACTURES UNDER LARGE STRAINS}

\section{Yu. I. Kadashevich, S.P. Pomytkin}

Saint-Petersburg State Technological University of Plant Polymers, 4, Ivan Chernykh str., Saint-Petersburg, 198095.

E-mail: sppom@yandex.ru

Endochronic constitutive relations in viscoplastic theory for large strain domain are studied. It is assumed that micro-fractures are initiated both in elastic and in nonelastic elements of deformed material. Two constitutive equations and two dilatation ones for elastic and non-elastic ranges are proposed. Dilatation parameters are introduced into strength condition for brittle and ductile fracture of materials.

Key words: non-elasticity, endochronic theory, large strains, constitutive equations, micro-fractures, strength condition.

Original article submitted 30/XII/2009; revision submitted 15/III/2010.

Yuliy I. Kadashevich (Dr. Sci. (Phys. \& Math.)), Professor, Dept. of Higher Mathematics. Sergey P. Pomytkin (Ph. D. (Phys. \& Math.)), Associate Professor, Dept. of Higher Mathematics. 